\title{
Ville intelligente et e-gouvernance en Inde, cartographier un nouveau paysage urbain
}

Smart city and e-governance in India, mapping a new urban landscape

Ciudad inteligente y gobierno electrónico, cartografíando un nuevo paisaje urbano

Éric Leclerc

\section{OpenEdition}

\section{Journals}

Édition électronique

URL : http://journals.openedition.org/mappemonde/4227

DOI : $10.4000 /$ mappemonde.4227

ISSN : 1769-7298

\section{Éditeur}

UMR ESPACE

\section{Référence électronique}

Éric Leclerc, "Ville intelligente et e-gouvernance en Inde, cartographier un nouveau paysage urbain »,

Mappemonde [En ligne], 128 | 2020, mis en ligne le 01 juillet 2020, consulté le 02 septembre 2020

URL : http://journals.openedition.org/mappemonde/4227 ; DOI : https://doi.org/10.4000/

mappemonde.4227

Ce document a été généré automatiquement le 2 septembre 2020

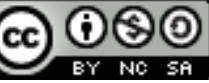

La revue Mappemonde est mise à disposition selon les termes de la Licence Creative Commons Attribution - Pas d'Utilisation Commerciale - Partage dans les Mêmes Conditions 4.0 International. 


\title{
Ville intelligente et e-gouvernance en Inde, cartographier un nouveau paysage urbain
}

\author{
Smart city and e-governance in India, mapping a new urban landscape \\ Ciudad inteligente y gobierno electrónico, cartografíando un nuevo paisaje \\ urbano
}

Éric Leclerc

\section{Introduction}

1 Dès son arrivée au pouvoir, le Premier ministre indien, Narendra Modi, a voulu concrétiser une de ses principales promesses électorales, construire 100 nouvelles villes intelligentes. Pour atteindre cet objectif, son gouvernement a lancé en juin 2015 «la mission villes intelligentes" (Smart City Mission - SCM). Celle-ci prévoit la construction de 100 villes intelligentes dans l'ensemble du pays soit par amélioration des infrastructures existantes, soit par création de villes-satellites ou de nouvelles villes. Dans cet article nous nous proposons d'analyser une dimension de cette nouvelle politique à travers l'exemple d'Amaravati, la future capitale de l'Andhra Pradesh. N. Chandrababu Naidu, le ministre en chef de cet État de l'Inde méridionale (figure 1) a choisi de construire une ville ex nihilo de grande ampleur $\left(217 \mathrm{~km}^{2}\right)$ avec 1,5 million d'habitants à l'horizon 2035, conçue sur le modèle global de la ville intelligente et durable (Upadhya, 2015; Leclerc, 2017). Amaravati est l'un des projets les plus ambitieux de la «mission villes intelligentes" et l'un des rares avec Rajkot et Navi Mumbay de création ex nihilo. Il adhère totalement à l'objectif «ville inclusive » de la SCM puisque N. C. Naidu a déclaré Amaravati " praja rajadhani », c'est à dire " capitale du peuple » en Hindi. Il compte y parvenir grâce aux Technologies de l'Information et de la Communication (TIC) afin d'améliorer la transparence du processus de construction, de disposer d'un système de suivi du projet efficace et de faire participer les citoyens à la production de la capitale. Ce dernier aspect montre que la ville 
intelligente ne se limite pas à une approche technologique, mais comporte également une dimension sociale et politique, comme l'écrit $\mathrm{P}$. Taraporevala à propos de la SCM «L'un des principaux moyens de légitimer la mission est la rhétorique de la participation citoyenne" (2018, p.11). Il semble donc légitime de questionner le mantra politique de «capitale du peuple » et le concept de ville intelligente mobilisés pour la future capitale, Amaravati. Notre objectif est d'analyser ce nouveau principe de gouvernance électronique afin de comprendre si les TIC pourront tenir leurs promesses d'une démocratie efficace et participative dans cette ville nouvelle.

Figure 1. Amaravati en Andhra Pradesh

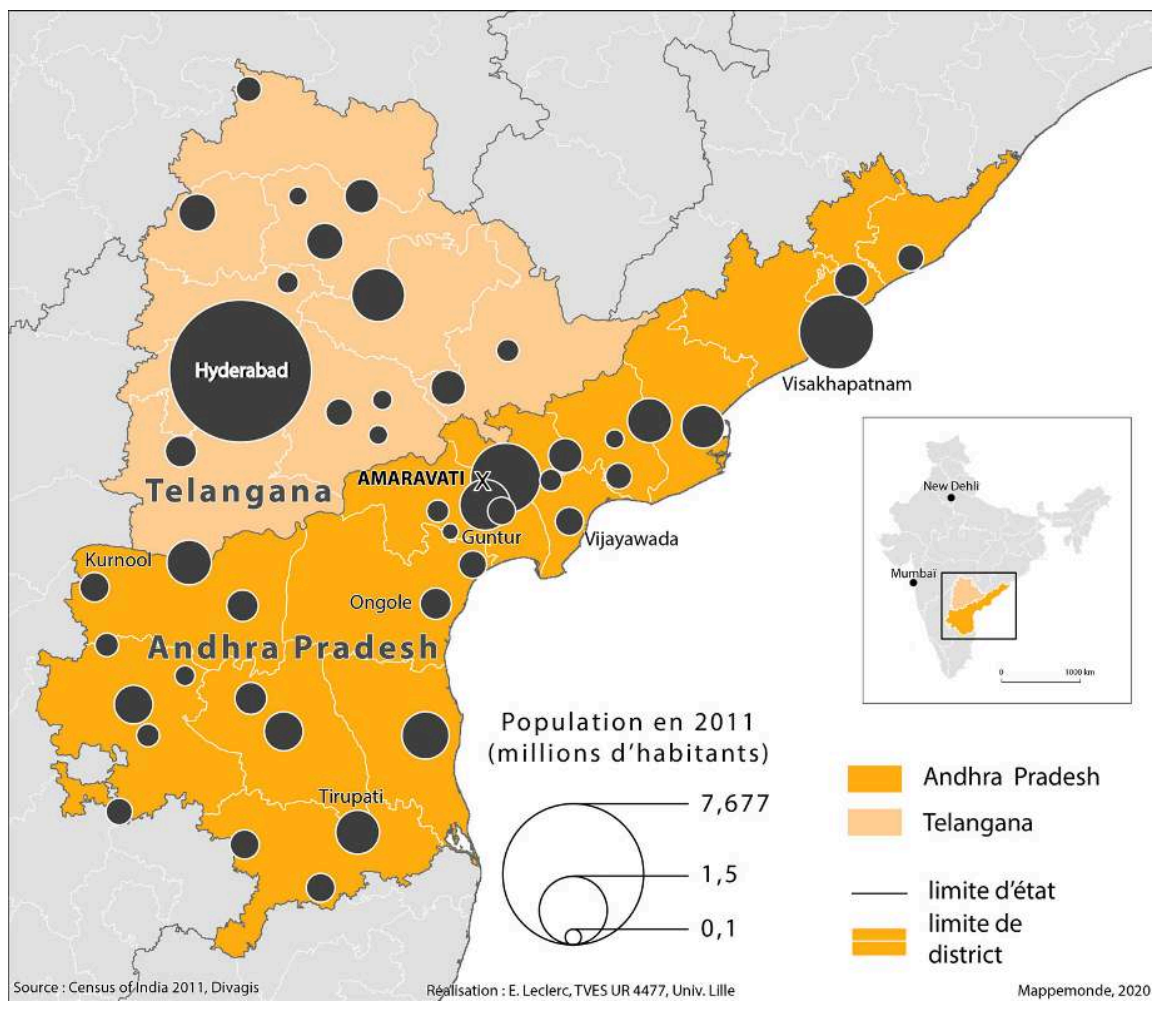

\section{Conception et gestion de la ville, une évolution parallèle face aux enjeux technologiques}

Les qualificatifs « numérique » et « intelligente » ont une signification très proche dans la littérature sur les villes. Les auteurs qui ont étudié l'évolution de la terminologie urbaine (Dameri et Cocchia, 2013, Joss, 2016) ont montré que le concept de "ville numérique " a été utilisé en premier dans les années 1990 au moment de l'émergence d'Internet. La ville numérique «s'appuie sur un environnement virtuel et sur les TIC (notamment Internet) pour améliorer la qualité de vie des citoyens grâce à la fourniture de services électroniques" (Dameri et Cocchia, 2013, p.4). Dans un premier temps, le recours au numérique se traduit par l'informatisation des données urbaines (cadastre, rôle d'imposition, état civil) pour parvenir à une gestion plus efficace des villes. Dans un second temps, la numérisation de l'administration permet de développer des services électroniques pour les citoyens. La notion de gouvernement 
électronique s'impose pour décrire cette réorganisation interne des services publics urbains au bénéfice des citadins.

En Inde, c'est après la création du ministère des Technologies de l'Information en 1999 qu'une politique de gouvernement électronique a été définie. Son objectif principal était de mobiliser les TIC au sein de l'administration pour s'affranchir du papier et proposer aux citoyens les premiers services en ligne. Dans le cas de l'Andhra Pradesh, cette politique s'est traduite par la mise en place de l'e-seva (service électronique) dès 1999. Dans la capitale de l'ancien Andhra Pradesh, Hyderabad (figure 1), ce nouveau modèle de gestion urbaine a pris forme sous le nom de Twin Cities Internet Services (TWINS). Grâce à la mise à disposition de kiosques électroniques, les citoyens pouvaient accéder à divers services gouvernementaux comme le paiement de leur facture d'électricité ou d'eau, le paiement de taxes à un guichet unique (Rao, 2002 ; Subhani et Kumar, 2013 ; Nalla, 2016). Après ce succès initial, le système a été déployé sous le nom d'e-seva dans la plupart des villes de l'Andhra Pradesh. Ce modèle de gestion urbaine électronique (e-gouvernement) repose sur une logique du gouvernement vers le citoyen (G2C Government to Citizen). C'est une approche descendante au service de la population. L'administration propose une offre sans forcément s'intéresser à la demande des citoyens.

4 Le deuxième concept, celui de "ville intelligente », prend de l'ampleur depuis une dizaine d'années avec la montée en puissance des réseaux sociaux, le Web 2.0 basé sur l'interaction directe entre les personnes. Le changement de qualificatif, de «numérique » à « intelligent » marque une nouvelle approche du modèle urbain et de sa gestion. Utilisée initialement aux États-Unis dans les années 1980 pour l'analyse urbaine, la «croissance intelligente » (smart growth) fait référence à la maîtrise par le contrôle et la planification de l'étalement urbain (Batty, 2013). Le concept est repris en 1992 lors de la conférence de Rio sur l'Environnement et le Développement, puis l'année suivante en Europe pour tenter de concilier les deux défis majeurs de l'époque, les TIC et l'environnement (Oural et al., 2018, p. 12). Il faut attendre la crise de 2008 pour que le qualificatif soit à nouveau associé à la ville dans le programme «smart city» lancé par IBM qui va populariser le concept. La définition de la «ville intelligente " n'en devient pas forcément plus facile tant elle recouvre des réalités différentes. Nous retiendrons celle proposée par A. Caragliu dès 2009 : « une ville est considérée comme intelligente lorsque les investissements dans le capital humain et social et l'infrastructure de communication classique (transport) et moderne (TIC) favorisent une croissance économique durable et une bonne qualité de vie, avec une gestion sage des ressources naturelles, dans le cadre d'une gouvernance participative " (Caragliu et al., 2009, p. 50 ; traduction, Meijer et al., 2016, p. 424). Cette définition montre que l'intelligence ne caractérise pas seulement les technologies, mais également les personnes qui les mettent en œuvre (capital humain). Elle implique aussi le déploiement d'un nouveau modèle de gestion des villes (gouvernance participative), une collaboration intelligente entre administration urbaine et citoyens. Enfin la "gestion sage des ressources naturelles » montre la généalogie de cette approche avec le développement durable défini dans les années 1990. Dans sa définition A. Caragliu conçoit la ville intelligente avec une approche ascendante, du citoyen vers le gouvernement (Citizen to Gouvernment $\mathrm{C} 2 \mathrm{G}$ ). Cette conception participative de la ville est une conséquence directe du Web 2.0. Elle montre la transition de la ville numérique et 
du gouvernement électronique (sans papier) à la ville intelligente et l'e-gouvernance dans laquelle les citoyens sont plus engagés dans la gestion de la ville.

En Inde le concept n'est pas aisé à définir comme l'avoue le site officiel de la SCM : "Même en Inde, il n'y a pas qu'une seule façon de définir la ville intelligente ${ }^{1}$ » et les premiers travaux sur cette mission n'ont pas tenté d'y remédier (Taraporevala, 2018; Khan, 2018). Amaravati qui a été retenue dans la liste des projets financés par le gouvernement central en juin 2016 revendique ouvertement le modèle de ville intelligente et durable. Le gouvernement de l'Andhra Pradesh a fait le pari de mobiliser la puissance des réseaux sociaux pour créer sa nouvelle capitale. Il utilise la force du Web 2.0 pour susciter l'adhésion des citoyens en faveur de son projet de développement. Il a créé de multiples plateformes pour renforcer l'engagement en ligne des citoyens : un compte Facebook (URL : http://facebook.com/prajarajadhani), un compte Twitter (avec les hashtags \#manaamaravati, \#manarajadhani respectivement \#monAmaravati et \#maCapitale) et une chaîne sur YouTube (youtube.com/prajarajadhani). La SCM a fixé comme objectif aux villes candidates d'utiliser une approche participative pour identifier avec les citoyens les principaux problèmes urbains à résoudre. Face à ce déploiement d'outils interactifs par le gouvernement d'Andhra Pradesh, nous nous interrogerons sur la finalité des autorités publiques. Les outils du Web 2.0 mobilisés autorisent-ils une réelle participation des citoyens dans la conception et la mise en œuvre du projet urbain? Amaravati sera-telle une ville plus inclusive grâce aux TIC?

\section{Méthodologie d'analyse du réseau de la gouvernance électronique d'Amaravati}

6 À la fin de l'année 2018 à Amaravati, cinq chantiers majeurs de construction sont en cours pour les logements des fonctionnaires, des parlementaires fédéraux et nationaux ainsi que la cour de justice. Le nouveau réseau routier avec son plan hippodamien a été tracé sur l'ensemble des $200 \mathrm{~km}^{2}$, mais son niveau d'achèvement est très variable. L'autoroute d'accès au noyau initial de la future capitale, l'axe monumental, son cœur politique, est terminé sur plus de $80 \%$ de sa longueur. Cependant, le peuplement d'Amaravati n'a pas encore commencé, si ce n'est pour les 100000 villageois qui habitaient cet espace avant le projet et qui en sont devenus, de facto, les premiers citadins. La nouvelle ville intelligente n'étant pas encore opérationnelle, il n'est pas possible de mesurer l'efficacité de sa gouvernance électronique dans la vie quotidienne. Par contre, nous pouvons analyser les différentes initiatives numériques lancées par l'État d'Andhra Pradesh pour mobiliser les citoyens autour de son projet de développement urbain. Leur recours aux plateformes du Web 2.0 indique une volonté de s'inscrire dans la logique de la ville intelligente (Dameri et al., 2014 ; Jucevicius et al., 2014), mais il faut déterminer le degré d'engagement des citoyens souhaité par le gouvernement d'Andhra Pradesh. Quel est le niveau de participation visé ? Sommesnous en présence d'une stratégie de communication mobilisant les TIC ou celles-ci sontelles mises en œuvre pour permettre une réelle collaboration des citadins à la conception d'Amaravati?

7 Pour obtenir le statut officiel de ville intelligente dans le cadre de la mission nationale (SCM), le gouvernement d'Andhra Pradesh a dû démontrer sa capacité à faire participer ses citoyens (Tarapolevala, 2018, 35). Le dossier de candidature d'Amaravati indique 
que leur compte Facebook avait en 2017 une audience de 3,6 millions d'utilisateurs, leur compte Twitter 400000 abonnés, et que leurs vidéos sur YouTube avaient étaient vues plus de 240000 fois (Smart city proposal, 2017, 103). Mais ces mesures quantitatives d'audience ne nous disent rien de l'engagement citoyen (intensité, forme). C'est pourquoi nous avons choisi une méthodologie fondée sur l'analyse de la structure des réseaux d'acteurs sur Internet pour évaluer le degré de participation des citoyens. En effet le projet de capitale concerne l'ensemble des habitants de l'Andhra Pradesh, voire au-delà, la diaspora telugu (langue parlée en Andhra Pradesh) puisque N. C. Naidu ambitionne de bâtir une ville de rang mondial. Pour mener cette enquête numérique, nous avons analysé les entités Web liées au projet d'Amaravati et effectué une étude structurelle (Diminescu, 2012) de ce réseau pour comparer l'influence respective des sites officiels et des autres acteurs sur le Web. Nous présenterons successivement les outils et la méthodologie d'analyse retenue pour construire un corpus d'entités, ainsi que les choix effectués pour constituer cet ensemble de données et révéler ce nouveau paysage urbain.

\section{Mobiliser un « robot orienté curation » Hyphe}

8 Inspirés par la méthodologie des humanités numériques, nous avons décidé d'utiliser le Web comme champ d'études (Ackland, 2013, Rogers 2013). Nous nous sommes appuyés sur des méthodes nativement numériques, tant pour les données que pour les méthodes en retenant trois des neuf approches identifiées par Rodgers: analyse des hyperliens, Facebook et Twitter. La méthode consiste à explorer le Web afin de découvrir tous les liens hypertextes entre les entités Web, il s'agit donc principalement d'une approche topologique couplée avec une analyse qualitative des liens. Pour collecter des entités, on utilise des robots qui fouillent le Web de façon systématique comme le fait Google pour alimenter son moteur de recherche. Cependant les robots entièrement automatiques ont plusieurs limitations. Leur utilisation de la méthode de la boule de neige pour explorer le Web crée des corpus très importants qui rendent le travail de post-traitement manuel très long. D'autres robots (IssueCrawler) sont liés à des moteurs de recherche (Rogers, 2010) et leurs résultats sont dépendants de l'algorithme de traitement de ce dernier (PageRank pour Google). C'est la raison pour laquelle nous avons choisi Hyphe, un robot semi-automatique adapté à la recherche en sciences sociales. C'est un outil de prospection du Web libre (http:// hyphe.medialab.sciences-po.fr/) développé au Medialab de Sciences-Po Paris (Jacomy et al., 2016). Ses créateurs ont conçu un outil qui permet de réaliser une fouille guidée du Web. Elle alterne des phases de recherche automatique (fouille) et des phases de sélection manuelle des résultats, c'est pourquoi ses développeurs définissent Hyphe comme un « robot orienté curation ». Hyphe est aussi un robot ouvert à la diversité du Web, il traite différents types d'entités (site Web, blog, ou réseaux sociaux, Facebook ou Twitter). Cet outil repose sur la construction d'un corpus autour d'une problématique de recherche. Dans notre cas, nous avons décidé d'explorer les entités Web liées à la construction d'Amaravati afin de découvrir les acteurs engagés sur Internet dans ce processus.

Nous avons procédé en trois étapes. Premièrement, nous avons construit une collection d'entités Web, notre corpus. Le point départ de la fouille a été constitué par des sites Web contenant le mot " amaravati », collectés grâce au moteur de recherche de Google. À partir de ce noyau initial d'entités, le robot de Hyphe a exploré tous les liens 
hypertextes de ces pages pour trouver les entités liées. C'est à l'issue de cette phase automatique que la curation de la collection commence en analysant et en sélectionnant les entités Web en lien avec notre problématique. Au cours d'un processus itératif (collecte entre le 4 juin et le 24 juillet 2017), nous avons exploré environ 4000 sites Web et obtenu un corpus final d'une centaine d'entités (98) reliées entre elles par 189 liens. Dans une seconde étape, nous avons spatialisé le réseau d'entités Web afin de pouvoir l'interpréter. Pour cette seconde phase, nous avons utilisé Gephi qui est un logiciel de visualisation de graphe de réseau libre (https:// gephi.org/). La troisième et dernière étape correspond à l'interprétation quantitative et qualitative du graphe. La structure du réseau révèle les relations entre les acteurs actifs sur le Web. Dans notre cas nous avons recherché les interactions entre les sites officiels du gouvernement d'Andhra Pradesh et la société civile. Une forte interconnexion serait un indice de la participation citoyenne. D'autre part, l'analyse qualitative du contenu des entités Web reliées permettra de connaître le type de relations développées, la nature de cette participation citoyenne autour d'Amaravati.

\section{Leçons tirées du processus de construction du corpus}

10 La construction du corpus par un processus de sélection piloté par le chercheur fournit déjà des indications intéressantes sur le contexte de notre sujet. Nous avons décidé de ne conserver que les entités Web en relation directe avec la nouvelle capitale Amaravati. C'est pourquoi nous avons éliminé de nombreux sites Web sur le bouddhisme, parmi eux des temples dans divers endroits du monde nommés Amaravati. Ils ne sont pas directement liés à la nouvelle capitale, mais à Amaravathi, la petite ville éponyme située à $15 \mathrm{~km}$ au nord-ouest du nouveau projet urbain. En reprenant le nom de l'ancienne capitale des Satavahanas, un royaume bouddhiste du III siècle av. J.-C. - III siècle apr. J.-C., le gouvernement d'Andhra Pradesh tire profit pour la nouvelle capitale de la notoriété religieuse et du passé politique de cette petite bourgade de 15000 habitants. Nous avons également éliminé du corpus les nombreux sites Web des musées archéologiques ou des galeries d'art liés au fameux stupa d'Amaravathi dont les bas-reliefs sont conservés au British Museum. Parmi tous les sites Web moissonnés consacrés à l'architecture, nous avons gardé seulement ceux qui mentionnaient le projet de la nouvelle capitale. Pour les sites des architectes, nous n'avons retenu que ceux impliqués à une étape du projet, le japonais Maki ou les Britanniques Rodgers et Foster. Le mot «Amaravati » est très présent sur le Web ce qui explique le nombre d'entités collectées par le robot (4000), mais elles font le plus souvent référence au glorieux passé d'un site archéologique proche plutôt qu'à la nouvelle capitale.

11 Le processus de curation de Hyphe permet d'effectuer une première analyse qualitative des entités Web en créant des catégories descriptives en lien avec notre problématique. $\mathrm{Au}$ fur et à mesure de l'exploration, nous avons identifié trois dimensions complémentaires des entités Web. Premièrement, nous avons créé une variable liée au type d'entité, site Web ou réseau social, en d'autres termes le Web 1.0 et le Web 2.0. À la dimension temporelle de cette distinction s'ajoute une mesure du degré d'interactivité (Web 1.0 conception descendante/Web 2.0 conception ascendante). La deuxième variable créée concerne l'identification du producteur des entités Web avec quatre catégories: gouvernement, entreprise privée, association ou initiative individuelle. Cette dimension permet de connaître les acteurs qui animent ce réseau. La troisième 
variable caractérise la dimension géographique affichée par les producteurs des entités Web avec trois catégories: Andhra Pradesh, Inde, Monde. L'identité spatiale peut-être explicite dans le titre du site Web ou sur les réseaux sociaux, mais lorsqu'elle est implicite, il faut lire leur contenu pour identifier l'échelle fédérale, nationale ou internationale de l'entité.

\section{Une approche descendante de la gouvernance électronique}

12 La méthodologie de construction et d'analyse du corpus des entités Web ne nous permet pas de suivre les acteurs dans le monde réel. Le terrain numérique que nous avons constitué autorise seulement l'analyse de liens entre des entités Web créées par des producteurs de site, blog ou réseau social. Le sens des liens est un objet de débat dans les humanités numériques ${ }^{2}$, ici il nous servira pour indiquer une volonté de participer à un réseau construit autour d'un objet, la capitale Amaravati. La direction des liens permettra d'identifier qui est à l'origine de cette relation. Nous procéderons à l'analyse structurale du réseau en quatre étapes: l'étude topologique du graphe (spatialisation) puis en comparant la distribution des trois variables qualitatives dans ce graphe.

\section{Analyse structurale du réseau des acteurs Web}

13 Comme indiqué précédemment, la spatialisation du graphe des entités Web a été réalisée avec le logiciel Gephi. Parmi les algorithmes proposés pour spatialiser le graphe, nous avons retenu celui développé par M. Jacomy dans le cadre du projet ediaspora, Force-Atlas. Celui-ci «simule un système physique afin de spatialiser un réseau. Les nœuds se repoussent comme des particules chargées, tandis que les arêtes attirent leurs nœuds, comme des ressorts " selon son auteur (Jacomy et al., 2014, p. 2). La structure produite ne dépend pas des variables attributaires des nœuds ou des liens, et la position d'un nœud dépend de l'ensemble des nœuds présents dans le graphe. Ce type d'algorithme est particulièrement bien adapté pour analyser les réseaux sociaux puisqu'une proximité structurale (topologique) se traduit par une proximité visuelle.

14 La spatialisation du graphe avec l'algorithme Force-Atlas permet une analyse à deux échelles: premièrement la position relative des entités Web dans l'espace total du graphe; deuxièmement le regroupement des entités en cluster dans certains sousensembles du graphe. Au premier niveau, l'algorithme construit une structure topologique qui répartit les nœuds en fonction du nombre et du sens de leurs liens. Notre corpus correspond à un graphe orienté puisque les liens hypertextes entre les entités ont chacun une origine et une destination. L'algorithme Force-Atlas privilégie les liens entrants identifiant ainsi les nœuds qui font autorité dans le réseau. Ces entités sont placées au centre du graphe. À l'inverse, les entités qui émettent beaucoup de liens sont définies comme des hubs (moyeux) et sont placées en périphérie. La centralité sur le graphe peut être interprétée d'autant plus facilement que la taille des nœuds qui représentent les entités Web ainsi que leur étiquette est proportionnelle au nombre de liens entrants. Ainsi, les grands cercles montrent les entités Web avec l'autorité maximale dans le réseau, les autres entités y font référence. À un second niveau, nous pouvons analyser sur le graphe les groupes d'entités Web qui constituent 
des agrégats (Ghitalla, 2004). Ils sont regroupés là aussi par l'algorithme Force-Atlas, car ils ont plus d'interconnexions entre eux qu'avec le reste du réseau.

Le graphe du réseau des acteurs Web liés à Amaravati peut donc être interprété à deux échelles, la position dans l'ensemble du graphe et deuxièmement l'emplacement des nœuds par rapport aux agrégats. Sur cette première visualisation du graphe (figure 2), nous avons identifié 5 agrégats de tailles inégales et organisés selon une répartition centre-périphérie. Le cluster principal situé au centre du graphe est constitué de sites Web avec deux autorités principales. Nous pouvons identifier par la taille du point et du nom de l'entité, en premier, un site Web du gouvernement de l'Andhra Pradesh (AP.gov.in) et en second, un site Web du gouvernement central (India.gov.in). Nous sommes donc en présence du Web institutionnel bien interconnecté. Sur la périphérie du graphe, nous trouvons quatre agrégats de comptes Facebook et Twitter. Le plus grand est créé par le compte Facebook de la capitale Amaravati, Praja Rajadhani (capitale du peuple). Il y a aussi deux groupes plus petits de comptes Facebook: le premier autour du compte officiel de N. C. Naidu en haut à gauche; le second, un peu au-dessus, est le compte d'Amaravati Voice, un média privé dédié à la capitale et à son développement. Il s'agit d'un moteur de recherche local destiné à compiler des informations sur la capitale et sa région qui fut actif de décembre 2015 à janvier 2018. Depuis cette date, les informations ne sont plus données qu'en Telugu par l'intermédiaire d'un compte Facebook. Chaque réseau social possède donc son propre cluster, car ils forment des groupes de comptes interconnectés entre eux, mais peu avec le reste du réseau. La position périphérique des agrégats des comptes Facebook du ministre en chef et d'Amaravati voice, s'explique par leur structure en hub. Un compte parent central a des liens sortants vers la déclinaison du même compte en d'autres langues. Les entités Web qui font autorité dans la structure topologique du réseau sont donc des sites institutionnels tant au niveau fédéral (Andhra Pradesh) que national (Inde). 
Figure 2. Structure du réseau des entités Web

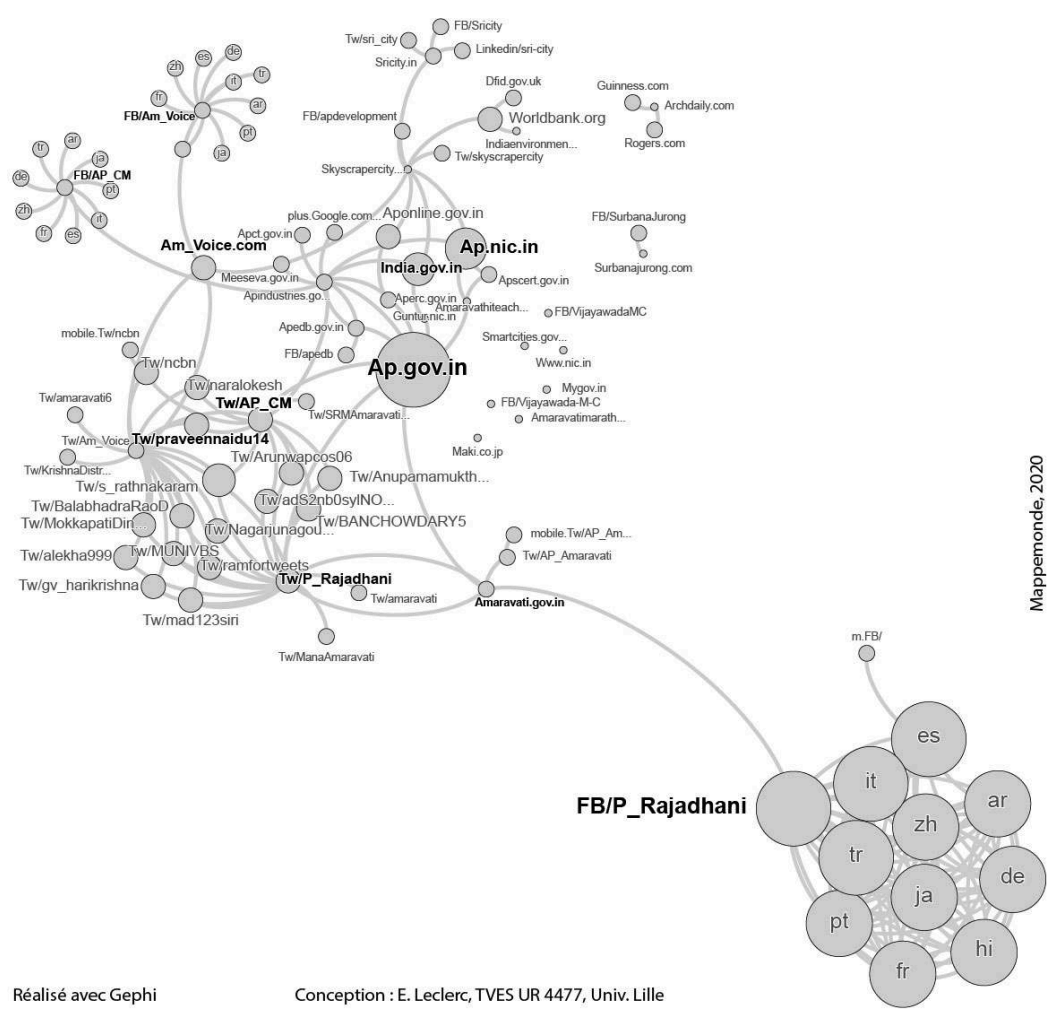

Source : Réalisé grâce à Hyphe

\section{Deux générations d'entités Web}

Pour comprendre la structure du graphe qui est indépendante des variables attributaires contenues dans les nœuds, il est possible de colorier ceux-ci et les liens en fonction des variables identifiées dans la phase de curation du corpus : le type d'entité, leur producteur et l'échelle spatiale revendiquée. Nous allons donc interpréter la structure du graphe en fonction de ces trois variables, en commençant par la dimension temporelle. L'organisation et la structure interne des agrégats sont assez différentes (figure 3) et s'interprètent facilement en fonction du type d'entité Web ici représenté par deux couleurs, le bleu pour le Web 1.0 et le rose pour le Web 2.0. Quantitativement, le Web de première génération compte le moins d'entités $29 \%$ de nœuds pour les sites contre $71 \%$ pour les réseaux sociaux. La spatialisation souligne aussi une seconde différence entre ces deux générations d'Internet. Les sites Web sont bien reliés entre eux, c'est pourquoi ils forment l'agrégat central du graphe. À l'inverse les réseaux sociaux se distinguent par leur fournisseur Facebook, Twitter. Ils forment des agrégats isolés les uns des autres et qui ne sont reliés au reste du graphe que par des sites Web classiques. Le cluster le plus important, Twitter, est composé de 3 pages de liens organisés autour des hashtags \#amaravati, \#ManaAmaravati et \#SRMAmaravatiLaunch et de 24 comptes Twitter. Il y a 7 comptes institutionnels : 2 pour la capitale (Praja rajadhani, Amaravati AP), 3 pour le parti au pouvoir (2 pour le ministre en chef, C. Naidu et Andhra Pradesh CM), 1 pour le district de Krishna (région d'implantation de la capitale) et 1 pour la ville de Sri City dans le sud de l'État. 
Figure 3. Les générations de Web d'Amaravti

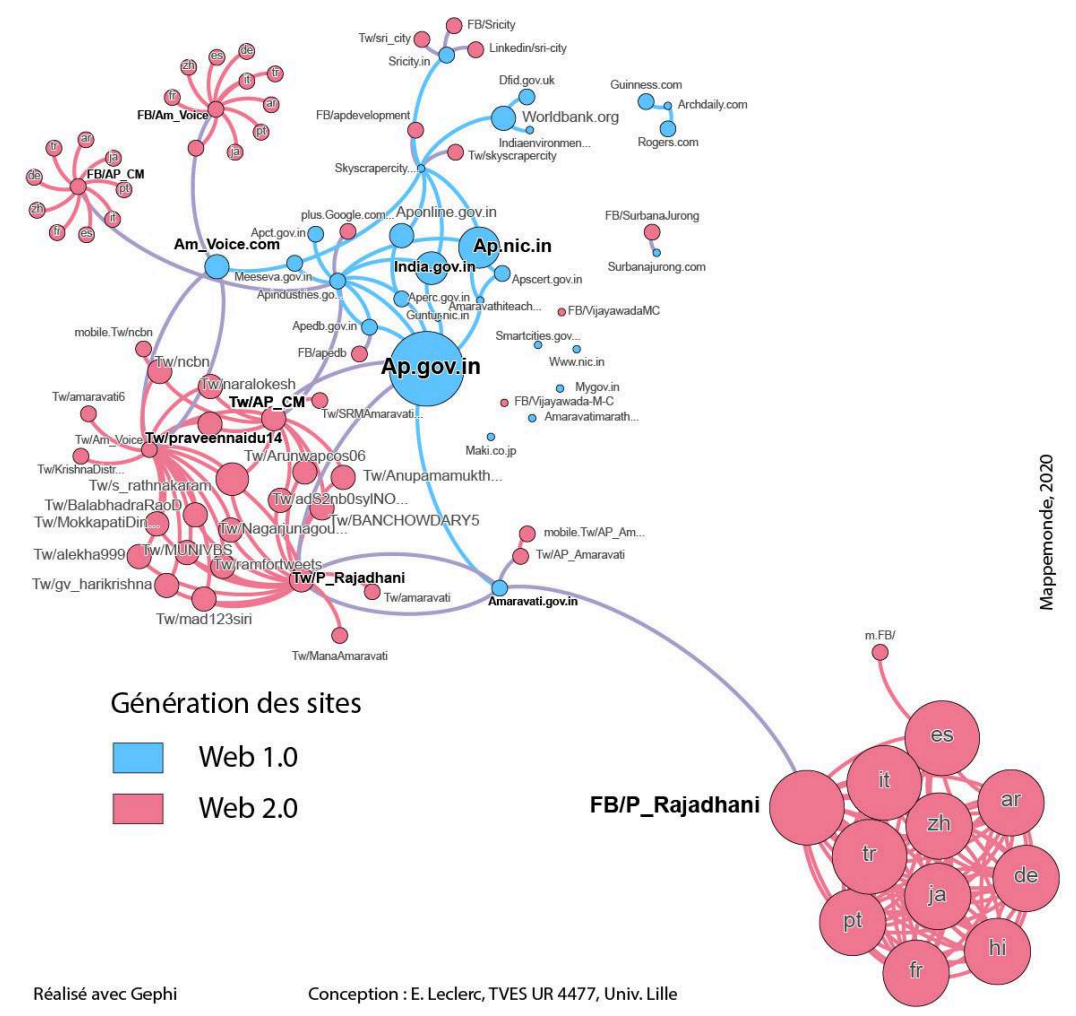

Source : Réalisé grâce à Hyphe http://hyphe.medialab.sciences-po.fr/

17 Il y a une différence majeure entre les deux réseaux sociaux, les comptes Twitter forment un cluster unique, alors que trois agrégats Facebook existent, le plus important autour du compte de la « capitale du peuple » Praja rajadhani, le second avec le compte du ministre en chef AP_CM et le dernier autour d'Amaravati voice. Cette différence provient du mode de fonctionnement des deux réseaux sociaux : le partage d'informations entre les comptes sur Twitter, alors que pour Facebook il faudrait pouvoir suivre les approbations (like) entre comptes pour analyser les liens. Cette diversité des réseaux sociaux explique probablement la volonté du gouvernement de l'Andhra Pradesh de multiplier le type de compte (Twitter et Facebook), et même d'ouvrir plusieurs comptes pour un même fournisseur (sur Facebook un compte pour la capitale et un pour C. Naidu, et deux fois plus sur Twitter) afin d'atteindre la plus large audience. Le type d'entité Web reflète l'évolution d'Internet avec un changement de génération des sites vers les réseaux sociaux, ces derniers permettant une plus grande interaction des acteurs. Les autorités gouvernementales dans l'État s'adaptent à cette transformation en investissant les deux types d'outils de communication.

\section{Un réseau de sponsors étatiques}

Sur la troisième visualisation (figure 4), la structure du graphe est identique, seules les couleurs des nœuds et des arêtes changent. Elles correspondent cette fois au type de producteur des entités Web. La moitié (54\%) des entités Web sont des sites institutionnels réalisés par des agences gouvernementales (en violet) principalement pour le Web 1.0 comme le montre le cluster central. Mais elles sont également actives sur le Web 2.0. Un agrégat à la gauche du graphe correspond au compte Facebook de C. 
Naiduet, l'autre en bas à droite à la capitale. Les institutions politiques sont aussi présentes sur Twitter ( $1 / 3$ de l'échantillon). Elles se distinguent par leur grand nombre d'abonnés, 4,2 millions pour le ministre en chef $\mathrm{C}$. Naidu, 0,6 million pour son fils Nara Lokesh qui est membre du gouvernement. Quel que soit le support utilisé, la logique reste pourtant la même, une communication descendante du gouvernement vers les citoyens (G2C). Les entreprises privées sont aussi très présentes sur ce graphe, en seconde position en nombre d'entités créées (en vert, $25 \%$ ), mais leur structure est totalement différente. Elles sont à la périphérie du graphe, soit car elles constituent des hubs (cluster Amaravati voice), soit parce qu'elles correspondent à des entités Web isolées. Il s'agit des sites des architectes impliqués dans le projet (Maki, Rogers) et de Surbana Jurong, une compagnie de Singapour qui a réalisé le plan directeur de la capitale. Ces entités ne sont pas connectées au réseau principal et très faiblement entre elles, ce ne sont pas les acteurs principaux de ce réseau.

Le troisième type de producteur correspond aux acteurs individuels (en bleu, 16 \%) qui sont présents uniquement dans les comptes Twitter au nombre de 27. Les deux tiers se présentent comme des comptes individuels, il s'agit d'un échantillon de personnes qui redistribuent des informations à propos de la capitale. Deux comptes ont été fermés depuis la collecte et 3 autres toujours ouverts ne sont plus actifs (1 depuis 2016 et 2 depuis 2018). On peut douter du caractère individuel de certains comptes au nombre de tweets envoyés. 3 comptes dépassent les 20000 tweets, alors qu'un compte individuel atteint difficilement 1500 tweets avec une forte activité. Il s'agit de partisans du Telugu Desam, le parti du ministre en chef au pouvoir C. Naidu qui participent à l'effort de communication de leur parti politique. Le profil des acteurs individuels, autant que leur profil public le laisse apparaître, est très divers. En dehors des activistes politiques mentionnés ci-dessus, les titulaires des comptes peuvent résider en Andhra Pradesh (Hyderabad, Vijayawada, Tirupati, Vizakhapatnam), ailleurs en Inde (Pune au Maharashtra) ou dans la diaspora (Kuwait). Il n'est pas possible de suivre tous les comptes qui retwittent ces informations sans faire une analyse spécifique de la Twittosphère. Méthodologiquement, il devient difficile de comparer un lien hypertexte entre deux sites Web qui durent dans le temps, et des redirections de tweets qui changent en fonction de l'information. C'est une des limites de l'analyse temporelle des réseaux d'Internet. Comment évaluer le poids d'un lien, compté de façon identique dans la topologie du réseau, alors qu'il correspond à deux générations différentes du Web?

Les associations (en orange, $4 \%$ ) constituent le dernier type de producteur d'entités Web. Elles ne sont pas très actives sur ce réseau ou sans lien avec des entités Web officielles. Il s'agit de deux portails d'information, le premier de professeurs, le second sur l'environnement, et de 2 sites pour des passionnés des gratte-ciels et pour l'organisation du marathon d'Amaravati. En conclusion, les entités institutionnelles dominent très largement ce réseau à la fois sur le Web 1.0 et 2.0. Il y a très peu de liens entre le secteur public et le monde de l'entreprise. De la même façon, la société civile est peu présente que ce soit par le biais de comptes individuels (blog, réseau social) ou dans le monde associatif souvent bien représenté sur le Web. 
Figure 4. Le réseau d'Amaravati selon le producteur de l'entité Web

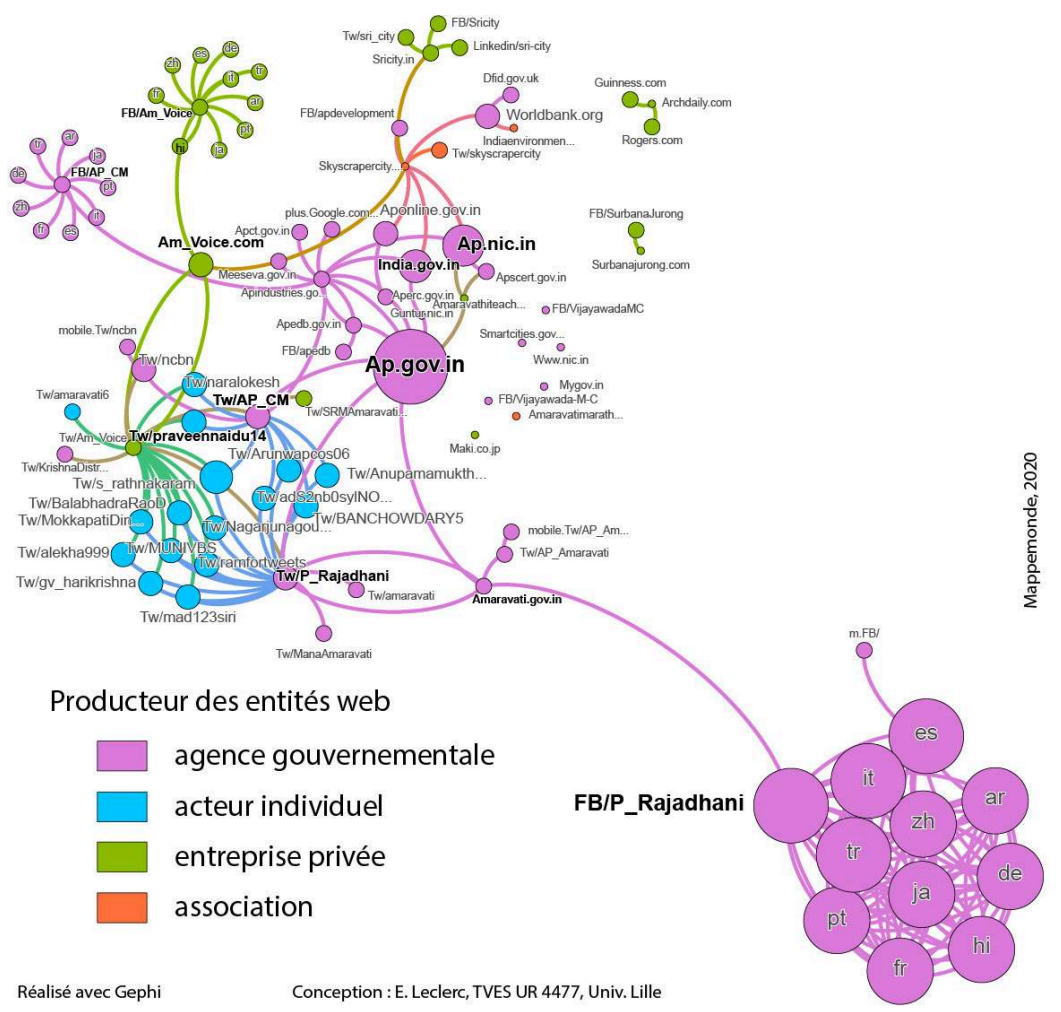

Source : Réalisé grâce à Hyphe

\section{Un réseau local}

La quatrième visualisation s'appuie sur la variable géographique. Chaque site ou compte sur un réseau social affiche une identité spatiale que nous avons classée en 3 échelles, fédérale, nationale et mondiale afin d'identifier l'aire d'influence potentielle des acteurs. Sur ce graphe (figure 5), chaque espace correspond à une couleur Andhra Pradesh (violet) pour le niveau fédéral, Inde (orange) pour le niveau national, et enfin le niveau mondial (vert). Le réseau des entités Web liées au projet d'Amaravati est numériquement dominé par l'Andhra Pradesh $(85 \%)$. Sa position périphérique correspond à une logique de réseaux sociaux. Ceci indique bien la stratégie des acteurs gouvernementaux qui ont fait le pari du Web 2.0 et recherchent un Internet plus participatif. Il y a donc une homogénéité des réseaux sociaux renforcée par l'affirmation de l'identité Andhra (échelle fédérale). À l'inverse, il y a une plus grande diversité du Web1.0 avec principalement deux échelles, nationale (site du gouvernement central de l'Inde) et mondiale (liens avec la banque mondiale ou l'agence britannique de développement DFID). L'agrégat central comprend tout de même un site d'autorité avec un portail Web du gouvernement de l'Andhra Pradesh qui sert de relais topologique entre les clusters Facebook et Twitter gouvernementaux. Cette stratégie de communication initiée par le gouvernement fédéral relève d'un véritable marketing territorial utilisant toutes les ressources d'Internet pour promouvoir la capitale avec une présence massive dans ce réseau, mais c'est aussi sa faiblesse. 
Figure 5. Les échelles géographiques du réseau d'Amaravati

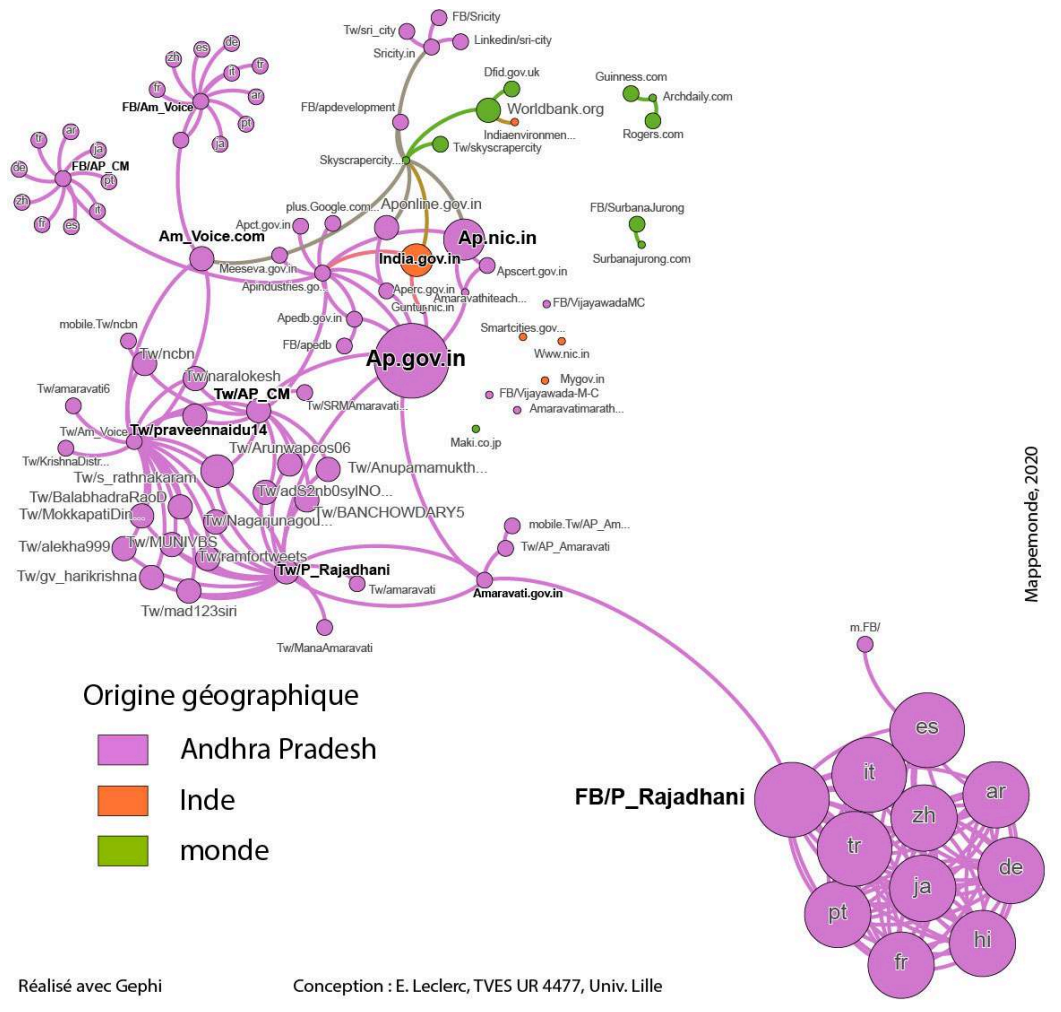

Source : Réalisé grâce à Hyphe

L'analyse de ce réseau montre qu'il a été initié par le gouvernement de l'Andhra Pradesh à la fois sur le Web 1.0 via le portail «Ap.gov.in " et sur les réseaux sociaux avec des comptes officiels sur Facebook ou Twitter. C'est globalement une approche descendante qui est en place, du gouvernement vers les citoyens. Par contre, on peut constater que cette partie du réseau n'est pas bien connectée au reste du Web. Les entreprises privées sont à l'écart et la plupart des liens de ce réseau sont avec d'autres entités publiques (gouvernement indien ou institutions internationales).

\section{Une conception très centralisée de la gouvernance électronique}

La construction d'Amaravati a débuté à la fin de l'année 2017 avec la construction des premiers logements pour les fonctionnaires sur le site. Les autres constructions réalisées sont deux universités privées SRM university à Nerukonda et VIT university à Nelapadu qui ont commencé leurs premiers cours en juin 2017. Dans ces circonstances, il ne sera pas possible de vérifier les promesses d'inclusion et de participation des citadins à la gestion de leur ville avant au moins une décennie, le temps de bâtir l'embryon d'une ville intelligente. C'est pourquoi nous avons décidé de mettre l'accent sur les outils TIC utilisés par ce gouvernement pour favoriser la création de la ville nouvelle. Au-delà des statistiques actuelles sur le nombre de visiteurs d'un site Web ou le nombre d'abonnés (followers) pour un réseau social, l'analyse structurelle du réseau d'entités Web fournit des indications plus consistantes. Nous avons pu démontrer premièrement que ce réseau est centré sur des sites institutionnels tant au niveau fédéral que national qui font autorité, deuxièmement que l'évolution d'Internet ne modifie pas fondamentalement cette organisation puisque les acteurs étatiques 
dominent le Web 1.0 comme le Web 2.0, troisièmement que ce réseau est produit par des acteurs locaux (appartenant à l'État fédéral). Dans notre cas, nous pouvons conclure que le système mis en place par le gouvernement d'Andhra Pradesh est un petit monde au sens sociologique. Les sites Web ou comptes sur les réseaux sociaux ne sont pas nombreux et ils sont fortement liés entre eux. Ils sont orientés pour une communication descendante, du gouvernement vers le citoyen et non l'inverse. La participation de ces derniers a été limitée à des votes sur des options architecturales pour quelques bâtiments de la future capitale comme la cour de justice ou le parlement. Par contre le plan directeur a été conçu par un cabinet d'experts de Singapour (Surbana Jurong) sans participation citoyenne.

Si nous ne disposons pas des statistiques de fréquentation des sites Web ou des réseaux sociaux, d'autres outils TIC mobilisés par le gouvernement d'Andhra Pradesh donnent des indications de leur usage. La volonté d'interaction avec les citadins s'est traduite par le développement d'une application «Mon Amaravati» (Mana Amaravati), «la première application de ce type en Inde qui réduit le fossé entre le gouvernement et ses concitoyens » comme l'indique son descriptif. L'application téléchargeable en version Androïd et iPhone (http://manaamaravatiapp.com/home) permet d'accéder aux services sociaux proposés par le projet et aux paysans de vendre leurs terres en ligne. Grâce à Internet, les paysans peuvent trouver des acheteurs partout dans le monde, et ces derniers peuvent comparer les offres sur l'ensemble de la zone. Bien que l'application ait été téléchargée 39500 fois, son bilan est très faible en matière de transactions foncières. Une centaine de ventes ont été réalisées depuis la mise en ligne alors que près de 65000 lots ont été redistribués aux paysans. La volonté de transformer la vie des citoyens grâce aux TIC lorsqu'elle existe se heurte à de nombreux obstacles tant techniques (qualité du réseau), cognitifs (fracture numérique) que sociaux (agents immobiliers).

\section{Conclusion}

Évaluer l'inclusivité d'une politique publique est une tâche difficile, et encore plus lorsque l'on travaille sur un projet urbain qui n'est pas encore réalisé comme Amaravati ou une décision récente comme la Smart City Mission. Au niveau national indien, les premières analyses de la SCM (Taraporevala, 2018) portent sur les projets annoncés plus que sur les résultats. D'ores et déjà, il apparaît que les budgets prévus pour les TIC n'atteignent pas $4 \%$ du montant total des investissements prévus par les villes (idem, graph. 26, p. 34) réduisant significativement le caractère «intelligent » de leur développement. Au niveau fédéral de l'Andhra Pradesh, l'exemple de la construction de la nouvelle capitale, Amaravati, démontre que si la stratégie du gouvernement a été très active sur le Web, la portée de sa gouvernance électronique n'est pas très ample. Même si ce gouvernement utilise le Web 2.0, c'est toujours dans une approche descendante et l'organisation de cette communication reste très centralisée. Nous pouvons considérer que ces outils sont plus au service de la construction d'une marque territoriale dans le but d'attirer les investisseurs que de la participation réelle des citoyens à l'élaboration et la réalisation du projet d'une capitale intelligente et durable. 


\section{BIBLIOGRAPHIE}

The Smart City Challenge Stage 2: Smart City Proposal. Amaravati the people's capital of Andhra Pradesh (2017). New Delhi, Smart City Mission, 251 p.

ACKLAND R. (2013). Web social sciences: concepts, data and tools for social scientists in a digital age. Thousand Oaks, CA : SAGE Publications, $224 \mathrm{p}$.

BARATS C. (2013). Manuel d'analyse du Web en sciences humaines et sociales. Paris : A. Colin, 258 p.

BATTY M. (2013). “Big data, smart cities and city planning”. Dialogues in Human Geography, vol. 3, nº 3, p. 274-279.

CARAGLIU A., DEL BO C., NIJKAMP P. (2009). "Smart Cities in Europe". In Proceedings to the 3rd Central European Conference on Regional Science. Košice, Slovaquie : Université technique de Košice, p. 16. DAMERI R. P., COCCHIA A. (2013). "Smart City and Digital City: Twenty Years of Terminology Evolution". In itAIS, X Conference of the Italian Chapter of AIS, "Empowering society through digital innovations", Milan : Université Bocconi, p. 1-8.

DAMERI R. P., ROSENTHAL-SABRouX C. (2014). Smart City: How to Create Public and Economic Value with High Technology in Urban Space. Springer Suisse, $239 \mathrm{p}$.

DIMINESCU D. (2012). E-diasporas Atlas: exploration et cartographie des diasporas sur les réseaux numériques. Paris : Éditions de la Maison des Sciences de l'Homme, 35 p.

GHITALLA F. (2004). La géographie des agrégats de documents sur le web. En ligne : https:// ateliercartographie.files.wordpress.com/2011/04/geographiedesagregatsweb-2003.pdf

JACOMY M., GIRARD P., OOGHE-TABANOU B., VENTURINI T. (2016). "Hyphe, a curation-oriented approach to webcrawling for the social sciences". In Tenth International AAAI Conference on Web and Social Media (ICWSM 2016). 17-20 mai, Cologne, Allemagne, p. 595-598.

JACOMY M., VENTURINI T., HEYMANN S., BASTIAN M. (2014). “ForceAtlas2, A Continuous Graph Layout Algorithm for Handy Network Visualization designed for the Gephi software". PLoS ONE, vol. 9, $\mathrm{n}^{\circ}$ 6, p. 12 .

Joss S. (2016), "Smart city a regressive agenda?". In Society for the History of Technology Annual Meeting 2016, 22-26 June 2016, Singapour, p. 9.

JUCEVIČIUS R., PATAŠIENĖ I., PATAŠIUS M. (2014). “Digital Dimension of Smart City: Critical Analysis”. Procedia - Social and Behavioral Sciences, vol. 156, p. 146-150.

KHAN S., TARAPOREVALA P., ZÉRAH M.-H. (2018). « Les villes intelligentes indiennes : défis communs et diversification des trajectoires ». Flux, vol. 114, nº 4, p. 86-99.

LECLERC É. (2017). « Rêve de capitale en Andhra Pradesh (Inde), lorsque l'utopie urbaine est au pouvoir ». In LEDUCQ D., SCARWELL H. et INGALLINA P., Modèles de la ville durable en Asie, Bruxelles : Peter Lang, p. 129-145

MEIJER A., RODRÍGUEZ BOLÍVAR M.P. (2016). « La gouvernance des villes intelligentes. Analyse de la littérature sur la gouvernance urbaine intelligente ». Revue internationale des sciences administratives, vol. 82, $\mathrm{n}^{\circ}$ 2, p. 417-435.

NALLA S. (2016). "E-Governance In Andhra Pradesh: An Impact Study Of Mee Seva". International Journal of Multidisciplinary Advanced Research Trends, vol. 3, $\mathrm{n}^{\circ}$ 1, p. 129-138. 
OURAL A., EVENO E., DURAND-TORNARE F., VIDAL M. (2018). Vers un modèle français de villes intelligentes partagées. Rapport à Monsieur le Ministre de l'Europe et des Affaires étrangères, 219 p.

RAO S. N. (2002). Economic restructuring programme: Naidus developmental experiment in Andhra Pradesh, 1995 to 2001. Thèse, New Delhi : Université Jawaharlal-Nehru, Centre d'études politiques. p. 311.

ROGERS R. (2010). “Mapping Public Web Space with the Issuecrawler”. In BROSSARD C. et REBER B., Digital Cognitive Technologies: Epistemology and Knowledge Society, Londres : Wiley, p. 115-126.

ROGERS R. (2013). Digital methods. Cambridge, Massachusetts : The MIT Press, 274 p.

SUBHANI S. R., KUMAR K. A. (2013). “E-Governance Initiatives In Andhra Pradesh". International Journal of Multidisciplinary Educational Research, vol. 2, n 1, p. 297-307.

TARAPOREVAlA P. (2018). Demystifying The Indian Smart City. An empirical reading of The Smart Cities Mission. Centre for Policy Research, $35 \mathrm{p}$.

UPADHYA C. (2017). “Amaravati and the New Andhra: Reterritorialization of a Region”. Journal of South Asian Development, vol. 12, $\mathrm{n}^{\circ}$ 2, p. 177-202.

\section{NOTES}

1. "Even in India, there is no one way of defining a smart city ", site web de la Smart City Mission consulté le $02 / 11 / 2016$.

2. Les sociologues ont très tôt interrogé la valeur des liens sur le Web, indice d'une relation entre acteurs sociaux ou simple connexion fonctionnelle entre deux sites Web par Hypertexte. Pour sa part Richard Rogers les interprète comme « des actes d'associations, de non-association ou de dissociation » qui ont une valeur sociologique et politique (Rogers, 2008, p. 4, cité dans Barats, 2013, p. 238).

\section{RÉSUMÉS}

La création d'une nouvelle capitale en Andhra Pradesh, Amaravati, est l'occasion de tester de nouvelles formes de participation citoyenne. Ce projet de «capitale du peuple » doit permettre de bâtir une des 100 nouvelles villes intelligentes indiennes en promouvant une e-gouvernance mobilisant les TIC. L'enjeu est de passer d'une approche par le haut, du gouvernement vers le citoyen (e-gouvernement), à une approche par le bas, du citoyen vers son gouvernement (egouvernance) grâce au Web 2.0. À partir d'une analyse structurale des réseaux d'acteurs s'exprimant sur Internet, nous constaterons que la participation citoyenne est faible dans cette phase de construction de la ville nouvelle.

The creation of Amaravati, a new capital in Andhra Pradesh, is an opportunity to test new forms of citizen participation. The "People's Capital" will be one of India's 100 new smart cities using ICT to promote e-governance. The challenge is to flip the top-down approach, government to the people, (e-government) into a bottom-up (citizen to government) situation with citizens (e- 
governance) using Web 2.0. The structural analysis of networks of actors using the Web reveals weak citizen participation in the current phase of construction.

La creación de la nueva capital en Andhra Pradesh, Amaravati, es una oportunidad para ensayar nuevas formas de participación ciudadana. La "Capital del Pueblo" es una de las cien nuevas ciudades inteligentes que se están impulsando en la India, y en donde se fomenta la gobernanza electrónica mediante las Tecnologías de la Información y Comunicación. El desafío es pasar de un enfoque desde arriba -del gobierno al ciudadano (gobierno electrónico)-, a un enfoque de abajo hacia arriba -del ciudadano a su gobierno (gobierno electrónico)-, a través de la Web 2.0. En esta fase todavía de construcción de la nueva ciudad, el análisis de las redes de actores que utilizan Internet revela que la participación ciudadana es todavía escasa.

\section{INDEX}

Thèmes: Enjeux de l'information territoriale locale ouverte et participative

Palabras claves : gobierno electrónico, gobernanza electrónica, redes sociales, ciudad inteligente

Keywords : e-government, e-governance, social networks, smart city

Mots-clés : e-gouvernement, e-gouvernance, réseaux sociaux, ville intelligente

\section{AUTEUR}

\section{ÉRIC LECLERC}

Univ. Lille, Univ. Littoral Côte d'Opale, ULR 4477 - TVES - Territoires Villes Environnement \& Société, F-59000 Lille, France 